\title{
In Situ TEM Investigation on the Effect of Atmosphere on Anatase-To-Rutile Phase Transformation
}

\author{
Hessam Ghassemi ${ }^{1}$, Wayne Harlow ${ }^{1}$, Rasit $\mathrm{Koc}^{2}$, and Mitra L. Taheri ${ }^{1}$
}

1. Department of Materials Science and Engineering, Drexel University, 3141 Chestnut St., Philadelphia, PA, 19140

2. Department of Mechanical Engineering and Energy Processes, Southern Illinois University, Carbondale, IL 62901

This research investigates the effect of atmosphere and temperature on the kinetics of the anatase-torutile phase transformation in titanium dioxide $\left(\mathrm{TiO}_{2}\right)$ nanoparticles using an in situ gas cell in a transmission electron microscope (TEM). High-resolution (HR) TEM images and diffraction patterns were captured in various gaseous environments (argon, oxygen, and nitrogen) and at different annealing rates up to $1000^{\circ} \mathrm{C}$ in situ to study the transformation phenomenon.

Titanium dioxide has several applications in fields such as photocatalyst, lithium-ion batteries and biotechnology. It forms three main polymorphs which include rutile, anatase and brookite. Rutile is the most stable form of $\mathrm{TiO}_{2}$ and the rest are considered metastable. Anatase, the second most important polymorph, stabilizes by converting to rutile at a wide range of temperature, which can be affected by oxygen defect level or presence of dopants ${ }^{1}$. Therefore, understanding the nature of this transformation as well as the effect of atmosphere on the kinetic is important to further develop their applications.

Various studies have been performed to characterize and understand the anatase-to-rutile phase transformation. Gouma and $\mathrm{Mills}^{2}$ have shown that anatase nanoparticles transformed to rutile and agglomerated to bigger particles after several hours of heat treatment at $1000^{\circ} \mathrm{C}$ in an air atmosphere. However, their post-treatment analysis lacks investigation of any possible intermediate mechanism. Joule heating experiments inside TEM were conducted to observe and characterize the phase transformation $^{3}$. These results revealed agglomeration of nanoparticles upon transforming of anatase to rutile phase. However, the experiments were only conducted under the vacuum of the TEM column, which lacks the effect of the type of the atmosphere on the phenomenon. Moreover, accurate measurements of temperature range upon which the transformation initiates, as well as the effects of atmosphere on this range need further investigation.

Our experiments were carried out using a Protochips ${ }^{\mathrm{TM}}$ in situ gas flow holder, shown schematically in Figure 1a, through which various kinds of gases can flow, and maintain an atmosphere of up to $1 \mathrm{~atm}$. The as-grown nanoparticles were $\mathrm{TiO}_{2}$ particles, both "bare" and coated with a nanoscale carbon shell. HRTEM images, shown in Figure 1b, confirm the anatase lattice structure of the initial product, with its Fast Fourier Transform shown in inset. The background in the images is the amorphous SiN windows of the cell. Three different kinds of sample including, pure $\mathrm{TiO}_{2}, \mathrm{TiO}_{2}-35 \mathrm{wt} \% \mathrm{C}$, and $\mathrm{TiO}_{2}-25 \mathrm{wt} \% \mathrm{C}$, were used to investigate the phase transition phenomenon. Atmospheres in this study included vacuum, nitrogen, oxygen and argon pure gases. The temperature was increased to $1000^{\circ} \mathrm{C}$, under different gas exposures, until the phase transition could be detected.

As indicated in Figure 2a, colonies of individual nanoparticles with diameters of 20-25nm are seen on the SiN electron-transparent window. The experiment was first carried out in an argon gas atmosphere, 
increasing the temperature from room temperature to $600^{\circ} \mathrm{C}$, in steps of $50^{\circ} \mathrm{C}$. As indicated with a red circle, four nanoparticles were initially in contact with each other, and upon increasing the temperature they started to coarsen, Figure $2 \mathrm{~b}$. However, their coarsening was stopped at $\sim 95 \mathrm{~s}$ of heat treatment, as illustrate in Figures $2 \mathrm{c}$ and $2 \mathrm{~d}$. In contrast, a separated colony of three particles, started to coarsen after longer period of heat treatment, indicated with white arrows.

In summary, this study investigated the anatase-to-rutile phase transition under exposure of different gases. It is shown than the agglomeration of rutile particles took place at the expense of neighboring anatase particles which resulted in elongated particles. This study can further develop the underlying mechanism of this phase transition in order to utilize their potential applications.

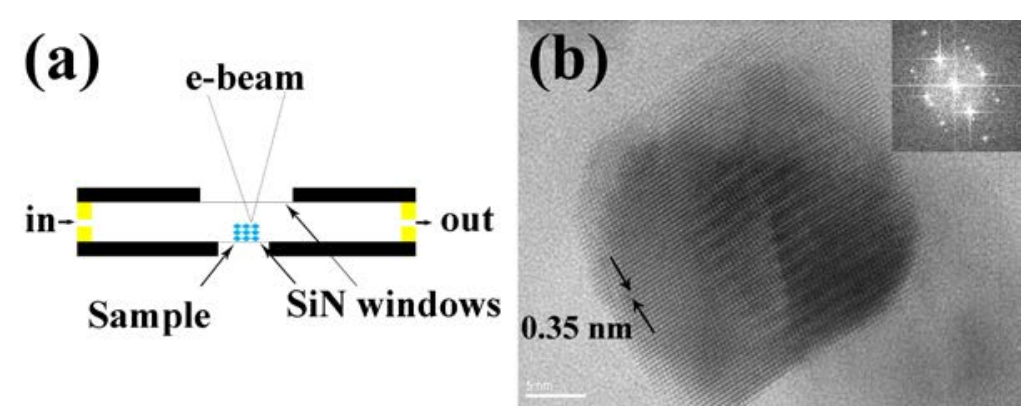

Figure 1. (a) Schematic of the sealed cell, where sample is placed between two electron-transparent $\mathrm{SiN}$ windows. Different gases can be introduced into the cell as indicted. (b) High-resolution TEM images of anatase nanoparticles placed inside a sealed cell, with the corresponding FFT as the inset. The background in the image is amorphous $\mathrm{SiN}$ window.
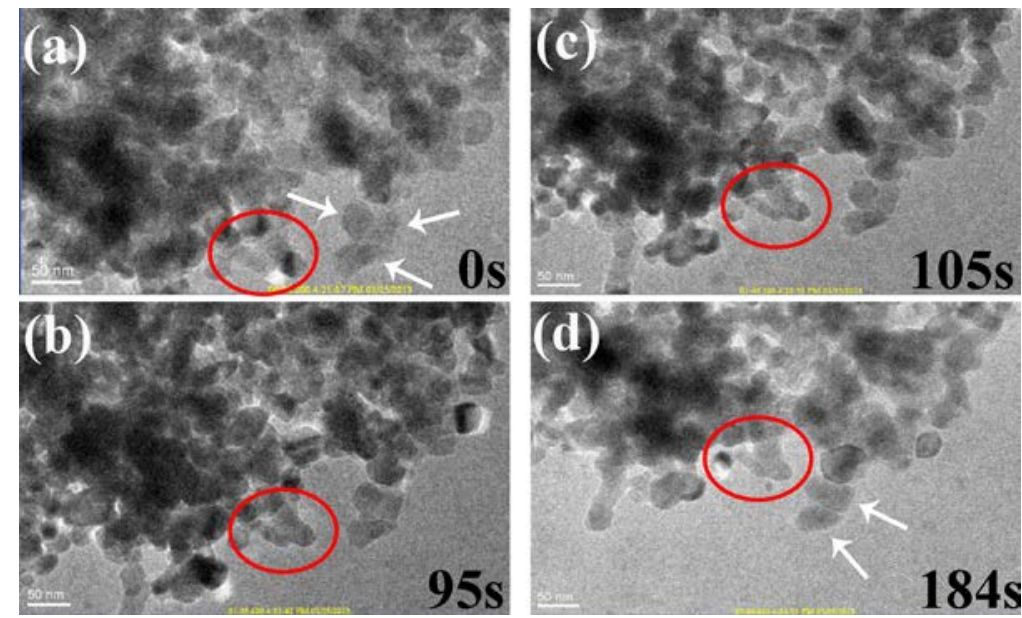

Figure 2. Phase transition under vacuum exposure where temperature was increased to $600^{\circ} \mathrm{C}$. (a) Represents the beginning (0s) condition, where two colonies of nanoparticles are marked with arrows and a red circle. (b-d) Illustrate phase transition of anatase nanoparticles to rutile and their coarsening.

References

1. D. H. Hanaor and C. Sorrell, J Mater Sci 46 (4), 855-874 (2011).

2. P. I. Gouma and M. J. Mills, Journal of the American Ceramic Society 84 (3), 619-622 (2001).

3. A. Asthana, T. Shokuhfar, Q. Gao, P. A. Heiden, C. Friedrich and R. S. Yassar, Advanced Science Letters 3 (4), 557-562 (2010). 